\title{
The content of fagopyrin and polyphenols in common and tartary buckwheat sprouts
}

\author{
SAMO KREFT ${ }^{1}$ \\ DAMJAN JANEŠ́2*,** \\ IVAN KREFT ${ }^{2}$ \\ ${ }^{1}$ Faculty of Pharmacy, University of \\ Ljubljana, SI-1000 Ljubljana, Slovenia \\ 2 Department of Agronomy, Biotechnical Facu \\ University of Ljubljana \\ SI-1000 Ljubljana, Slovenia
}

Accepted July 1, 2013
Dried buckwheat herb is used in medicinal products whereas fresh green plant parts, especially sprouts, are consumed as a vegetable. The herb contains fagopyrins, which cause sensitivity to light after ingestion. The aim of this study was to investigate the impact of different growing conditions and the development phase on the U⿴\zh11⿰一一千口, tent of fagopyrin and phenolic compounds in buckwheat sprouts. Total flavonoid and total phenol contents, fagopyrin content and antioxidant activity were determined spectrophotometrically. Fagopyrin and flavonoids were located almost exclusively in cotyledons. Based on a comparison to hypericin toxicity, the recommendable intake of buckwheat sprouts was estimated to be less than $40 \mathrm{~g}$ per day.

Keywords: buckwheat, Fagopyrum (Polygonaceae), fagopyrin, polyphenols, sprouts, phototoxicity

Common buckwheat (Fagopyrum esculentum) is an important crop due to its nutritious seeds. It is similar to the rarely cultivated bitter tasting tartary buckwheat (Fagopyrum tataricum). Its herb is used in herbal medicinal products, for green buckwheat tea, for producing buckwheat green leaf flour as an additive to some food products, while fresh green plant parts are consumed as a vegetable $(1,2)$. Buckwheat herb is especially known as a rich source of flavonoids $(2,3)$ and tannins $(4,5)$. Buckwheat herb tea was shown to protect from leg oedema in patients with chronic venous insufficiency in a randomized, double blind, placebo-controlled clinical trial (6). The use of buckwheat herb is recommended for the prophylaxis and treatment of diabetic patients suffering from retinopathy (7). Four different flavonoids [rutin (2.57 $\mathrm{mg} \mathrm{g}^{-1}$ dry mass), isoorientin $(1.27 \mathrm{mg}$ $\mathrm{g}^{-1}$ dry mass), vitexin (0.11 $\mathrm{mg} \mathrm{g}^{-1}$ dry mass) and isovitexin (0.04 $\mathrm{mg} \mathrm{g}^{-1}$ dry mass)] (8) and four anthocyanins [cyanidin 3-O-glucoside ( 0.16 to $0.20 \mathrm{mg} \mathrm{g}^{-1}$ dry mass), cyanidin 3-O-rutinoside (5.55 to $6.57 \mathrm{mg} \mathrm{g}^{-1}$ dry mass), [cyanidin 3-O-galactoside, and cyanidin

\footnotetext{
* Correspondence; e-mail: damjan.janes@ffa.uni-lj.si

** Partial results of this research were presented at 59th International Congress and Annual Meeting of the Society for Medicinal Plant and Natural Product Research in Antalya, Turkey, in 2011.
} 
3-O-galactopyranosyl-rhamnoside] were found in buckwheat sprouts (9). Besides polyphenols with a high antioxidative potential, buckwheat herb also contains fagopyrins, a group of substances similar to hypericin of St John's wort (Hypericum perforatum), which cause sensitivity to light after ingestion of large amounts of green parts of buckwheat (1). Buckwheat herb originally contains protofagopyrin, which converts into fagopyrin and other similar substances after exposure to daylight $(10,11)$. Quinones, like hypericin and fagopyrin, express a light-dependent activity; they may be used in medicine as potential sensitizers in photodynamic therapy (12). Literature data on the fagopyrin content in buckwheat leaves, stems and flowers are very scarce and there is no data on its content in buckwheat sprouts. Our previous studies and other studies showed that leaves of common buckwheat contain 0.4 to $0.6 \mathrm{mg} \mathrm{g}^{-1}$ dry mass, stems 0.04 to $0.12 \mathrm{mg} \mathrm{g}^{-1}$ dry mass and flowers $0.64 \mathrm{mg} \mathrm{g}^{-1}$ dry mass of fagopyrin. No fagopyrin was detected in buckwheat groats and the hulls contain only $0.02 \mathrm{mg} \mathrm{g}^{-1}$ dry mass $(11,13)$.

Fagopyrin was found in vitro to be less phototoxic than hypericin (14). At a daily dose of $5.6 \mathrm{mg}$ for 15 consecutive days and a single dose of $11.25 \mathrm{mg}$ of hypericin, the UV light sensitivity of human volunteers was only marginally increased (15). Severe cutaneous phototoxicity of hypericin was observed at a dose of $0.5 \mathrm{mg} \mathrm{kg}^{-1}$ daily $(35 \mathrm{mg}$ per day for a $70 \mathrm{~kg}$ adult man) (16). Tests in rats or mice showed that a single dose of $1.25 \mathrm{~g} \mathrm{~kg}^{-1}$ of dried buckwheat flowers had no effect, a dose of $2.5 \mathrm{~g} \mathrm{~kg}^{-1}$ produced slight but distinct sensitivity, and the reaction was severe at doses between 5 and $10 \mathrm{~g} \mathrm{~kg}^{-1}$ (17).

The aim of this study was to investigate the impact of different growing conditions and development phases on the content of phenolic substances and fagopyrin in buckwheat sprouts and to estimate the safe daily intake of buckwheat sprouts.

\section{EXPERIMENTAL}

\section{Reagents}

Folin-Ciocalteau (FC) and 2,2-diphenyl-1-picrylhydrazyl (DPPH) reagents were supplied by Fluka (Switzerland). Aluminium(III) chloride hexahydrate, sodium carbonate anhydrous, ethanol $96 \%$, methanol and tetrahydrofuran were purchased from Merck (Germany). Pyrogallol was supplied by Sigma-Aldrich (Germany), rutin and hypericin were purchased from Carl Roth (Germany).

\section{Instruments}

Buckwheat sprouts were grown in a Freshlife Automatic Sprouter Model 2000 supplied by Tribest Corporation (USA). Plant material was lyophilized using LIO-2000 apparatus produced by Kambič (Slovenia). SpeedVac ${ }^{\circledR}$ DNA 110 system produced by Savant (USA) was used for preparation of dry extracts by evaporation under reduced pressure. Ultrasonic bath Sonorex Digitec DT 103 H was supplied by Bandelin (Germany) and the centrifuge Centric 150 with rotor RA24M was produced by Tehtnica (Slovenia). Spectrophotometrical measurements were performed using a Safire microplate reader supplied by Tecan (Switzerland). 


\section{Preparation of plant material}

Common buckwheat [Fagopyrum esculentum Moench (Polygonaceae)], cv. Darja seeds of $0.026 \mathrm{~g}$ mass and tartary buckwheat [Fagopyrum tataricum (L.) Gaertn.] seeds of a domestic variety from Luxembourg were sown for sprouts production. In the sprouter, the seeds were positioned on a perforated plastic plate and automatically watered every $30 \mathrm{~min}$. Excess water flowed through the perforation. One experiment was performed for comparison by sowing the seeds on a non-perforated plate (Petri dish) and watered once daily. Other growing parameters (time, light) are presented in the Results and discussion section. After the harvest, the plants were lyophilized at $-40{ }^{\circ} \mathrm{C}$ and $0.1 \mathrm{~Pa}$.

\section{Determination of phenolic compounds}

Total flavonoid and total phenol contents and antioxidant activity were determined spectrophotometrically using $\mathrm{AlCl}_{3}, \mathrm{FC}$ and $\mathrm{DPPH}$, respectively, as previously reported (3). Briefly, $200 \mathrm{mg}$ of a powdered buckwheat sample was extracted with $10 \mathrm{~mL}$ of $60 \%$ ethanol overnight on a shaker. The mixture was then centrifuged at $4000 \mathrm{rpm}$ for $10 \mathrm{~min}$ and the clear solution was used for determination of flavonoids and total phenols. The reagent-free blanks were used, since the samples absorb light at specified wavelengths, but the reagents do not.

For determination of flavonoids, two aliquots of each $180 \mu \mathrm{L}$ of the sample were prepared. Twenty microlitres of $5 \% \mathrm{AlCl}_{3}$ in methanol was added to the first aliquot and $20 \mu \mathrm{L}$ of methanol was added to the second aliquot. After $30 \mathrm{~min}$, absorbance of both solutions was measured at $425 \mathrm{~nm}$. The concentration was calculated from the differences between the measurements and by comparison to a standard solution of rutin $(0.02 \mathrm{mg}$ $\left.\mathrm{mL}^{-1}\right)$.

For determination of total phenols, two aliquots of each $20 \mu \mathrm{L}$ of the sample were prepared. One hundred and fifty microlitres of water and $10 \mu \mathrm{L}$ of FC reagent were added to the first aliquot and $160 \mu \mathrm{L}$ of water was added to the second aliquot. After $3 \mathrm{~min}, 20$ $\mu \mathrm{L}$ of $20 \% \mathrm{Na}_{2} \mathrm{CO}_{3}$ in water was added to both aliquots. After $60 \mathrm{~min}$, absorbance of both solutions was measured at $750 \mathrm{~nm}$. The concentration was calculated from the differences between the measurements and by comparison to a standard solution of pyrogallol $\left(0.1 \mathrm{mg} \mathrm{mL}^{-1}\right)$.

For determination of antioxidative activity, two aliquots with $100 \mu \mathrm{L}$ of the sample, were prepared (diluted with $60 \%$ ethanol if required). One hundred microlitres of DPPH solution ( $3.9 \mathrm{mg} \mathrm{mL}^{-1}$ of methanol) was added to the first aliquot and $100 \mu \mathrm{L}$ of methanol was added to the second aliquot. After $60 \mathrm{~min}$, the absorbance of both solutions was measured at $515 \mathrm{~nm}$. The concentration was calculated from the differences between the measurements and by comparison to a standard solution of pyrogallol $\left(0.1 \mathrm{mg} \mathrm{mL}^{-1}\right)$.

\section{Determination of fagopyrin}

The fagopyrin content was determined by a spectrophotometric method, as described in our previous study (13). This method was found to give slightly higher results compared to the recently developed HPLC method (11). Briefly, $200 \mathrm{mg}$ of powdered sample was extracted with $6 \mathrm{~mL}$ of $80 \%$ tetrahydrofuran in water at $65{ }^{\circ} \mathrm{C}$ for $30 \mathrm{~min}$. 
The sample was then centrifuged at $4000 \mathrm{rpm}$ for $10 \mathrm{~min}$. A clear solution was transferred into a new test tube and the sediment was extracted. After centrifugation, a clear solution was added to the solution from the first extraction. One millilitre of the combined extract was transferred to a plastic microcentrifuge tube and evaporated under reduced pressure. The dried extract was suspended in $500 \mu \mathrm{L}$ of methanol using an ultrasonic bath and then centrifuged at $12000 \mathrm{rpm}$ for $10 \mathrm{~min}$. Three hundred microlitres of clear solution was transferred to a microtiter plate vial and the absorbance was measured at $590 \mathrm{~nm}$. The concentration was calculated by comparison to a standard solution of hypericin $\left(0.02 \mathrm{mg} \mathrm{mL}^{-1}\right)$.

\section{Statistical analyses}

Each sample was analyzed tree times. Average and standard deviation were calculated and presented in figures as columns and error bars. The samples were compared by ANOVA and Student's $t$-tests.

\section{RESULTS AND DISCUSSION}

\section{Sprouting time}

The content of flavonoids, polyphenols and fagopyrin in buckwheat sprouts were ranged from 0.021 to $0.65,0.26$ to 1.5 and 0.0025 to $0.041 \%$ of dry mass, respectively. Fig. 1 shows that the content of polyphenols increased 2-fold in two weeks compared to the content of polyphenols at day 0 in the plate where the sprouts were watered only once a day. In the sprouter where the water conditions were optimal, the contents of phenols reached 2 to 4 times higher contents than in the plate. Differences between the two cultivation methods were significant at days 7 and 10 ( $t$-test: $p=0.003$ and 0.005 , respectively). The content of fagopyrin in the sprout increased 7 -fold in the plate and 14-fold in the sprouter. The differences between the two cultivation methods were significant at days 7 and 10 ( $t$-test: $p=0.002$ and 0.0005 , respectively). The content of fagopyrin in 14-day-old buckwheat sprouts grown in the sprouter was nearly the same as that reported for mature plants $(11,13)$, but the content of polyphenols was only approximately 20 to $30 \%$ of mature plants. All the contents are expressed per sprout dry mass.

Some increase in the content of secondary metabolites in the sprouts can theoretically be a consequence of decreased dry mass of an individual sprout compared to the seed. At the beginning of germination, respiration exceeds the photosynthesis and the amount of dry mass decreases. To test the importance of this phenomenon, the mass of 30 dry seeds and dry mass of 30 sprouts (with husks) were measured at each development stage. It was found that the mass of the sprout gradually decreased up to the tenth day, when it reached $65 \%$ of the original mass. Dry mass of the sprout increased afterwards (data not shown). 


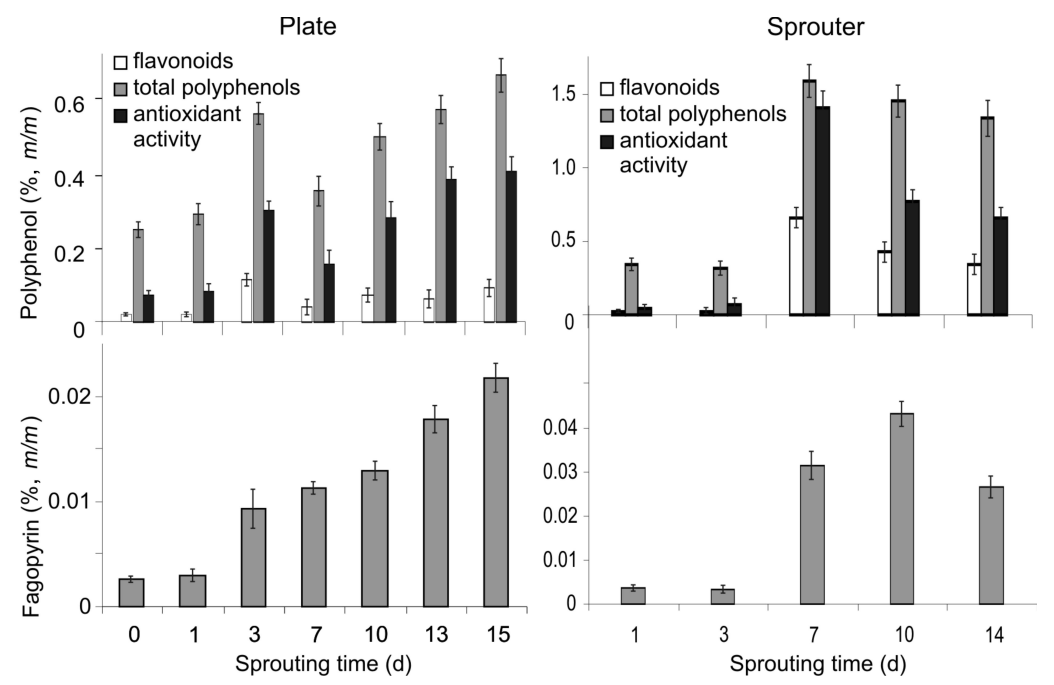

Fig. 1. The influence of sprouting time on polyphenols and fagopyrin in buckwheat sprouts growing in the sprouter (right) and in the plates (left). Mean $\pm \mathrm{SD}, n=3$.

\section{Distribution of substances in the sprout}

Plants for this experiment were harvested on the eighth day of sprouting. Fig. 2 shows there were no flavonoids in the roots, only small amounts in the stem and most of them in the cotyledons. The content of other polyphenols and total antioxidant activity in the roots was quite high (50\% compared to cotyledons). Fagopyrin was also located almost exclusively in cotyledons. The differences in flavonoids, total polyphenols, antioxidant activity and fagopyrin content between the organs were statistically significant (ANOVA: $p=0.001,0.012,0.18$ and 0.002, respectively). It is interesting to note that the content of flavonoids, polyphenols and fagopyrin in total sprouts in independent cultivation experiment (performed one month later), was very similar to the content obtai-

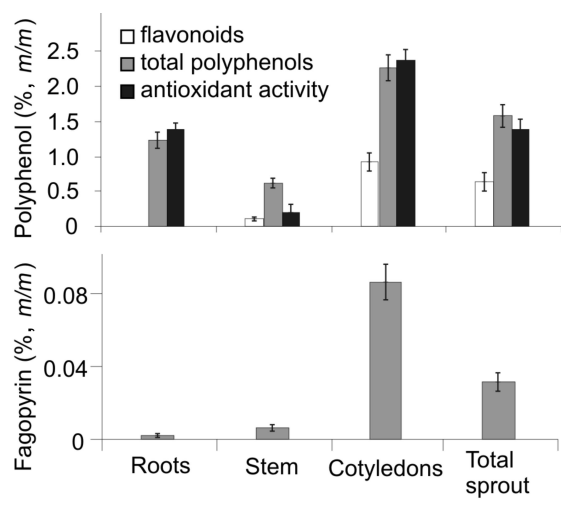

Fig. 2. Distribution of polyphenols and fagopyrin in sprout organs. 
ned in the sprouter (Fig. 1). Compared to the sprouts cultivated in the plate, the content of flavonoids, polyphenols and fagopyrin was 12-, 6- and 6-fold higher, respectively.

\section{Influence of illumination}

The plants for the experiment were grown in direct light (opened sprouter next to the window), in the shade (sprouter covered with an opaque gray plastic cover) and in the dark (sprouter cowered with aluminium foil). The content of flavonoids, total polyphenols, fagopyrin and antioxidant activity were measured. Fig. 3 shows that light exposure increased the content of flavonoids and fagopyrin more than 2-fold, but that it had no effect on the content of total polyphenols and on antioxidant activity (ANOVA: $p=$ $0.036,0.013,0.759$ and 0.846 , respectively).

Fig. 3. Influence of illumination on the content of metabolites (flavonoids, polyphenols, fagopyrin) and antioxidant activity in buckwheat sprouts.

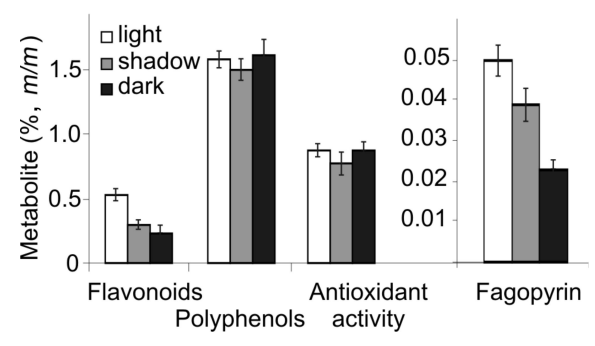

\section{Tartary buckwheat}

Sprouts of tartary buckwheat (10 days in the sprouter) contained an approximately 2-fold higher amount of all measured secondary metabolites than the corresponding sprouts of common buckwheat. The content of flavonoids was 1.1 to $1.4 \%$, polyphenols 2.0 to $3.3 \%$, antioxidant activity was equivalent to 2.2 to $3.6 \%$ of pyrogallol. This means that the antioxidant activity of buckwheat polyphenols is approximately equivalent to the antioxidant activity of pyrogallol. The content of fagopyrin was 0.10 to $0.12 \%$. All the differences were statistically significant ( $t$-test: $p=0.021, p=0.036, p=0.014$ and $p=$ 0.008 , respectively). It was previously found that the seeds of tartary buckwheat contained much higher amounts of flavonoids than common buckwheat (18), but the difference decreased during germination (19), which is in accord with our observations that the difference in the content of flavonoids in 10 days old sprouts of both species is only 2 to 3-fold in favour of tartary buckwheat.

\section{CONCLUSIONS}

The results of this research show that buckwheat sprouts can be used as a rich source of antioxidants in human nutrition. The ratio of desired antioxidants and undesired fagopyrin is however more favourable in fully grown plants than in sprouts. Since there is no accurate toxicological information on fagopyrin, safe intake of buckwheat sprouts 
can be estimated on the basis of hypericin phototoxic doses if further consideration of molecular differences (piperidinyl moieties) to hypericin is not done. Taking this into account, the consumption of $0.14 \mathrm{~g}$ of dry mass sprouts per $\mathrm{kg}$ body mass per day can be estimated, which corresponds to approximately 40 sprouts. The intake of $10 \mathrm{~g}$ of dry mass (or approximately $30 \mathrm{~g}$ of fresh mass) of buckwheat sprouts may, on the other hand, cause severe phototoxicity, but this might strongly depend on the exposure to sunlight, body mass and age.

Acknowledgements. - The skillful laboratory assistance of Maja Avguštin, Petra Banič and Ina Hudales is greatly appreciated.

\section{REFERENCES}

1. I. Hinneburg and R. H. H. Neubert, Influence of extraction parameters on the phytochemical characteristics of extracts from buckwheat (Fagopyrum esculentum) herb, J. Agric. Food Chem. 53 (2005) 3-7; DOI: 10.1021/jf049118f.

2. I. Kreft, N. Fabjan and K. Yasumoto, Rutin content in buckwheat (Fagopyrum esculentum Moench) food materials and products, Food Chem. 98 (2006) 508-512; DOI: 10.1016/j.foodchem.2005.05. 081.

3. S. Kreft, B. Štrukelj, A. Gaberščik and I. Kreft, Rutin in buckwheat herbs grown at different UV-B radiation levels: comparison of two UV spectrophotometric and an HPLC method, J. Exp. Bot. 53 (2002) 1801-1804; DOI: 10.1093/jxb/erf032.

4. J. Kalinova, J. Triska and N. Vrchotova, Distribution of vitamin E, squalene, epicatechin and rutin in common buckwheat plants (Fagopyrum esculentum Moench), J. Agric. Food Chem. 54 (2006) 5330-5335; DOI: 10.1021/jf060521r.

5. J. Kalinova and N. Vrchotova, Level of catechin, myricetin, quercetin and isoquercitrin in buckwheat (Fagopyrum esculentum Moench), changes of their levels during vegetation and their effect on the growth of selected weeds, J. Agric. Food Chem. 57 (2009) 2719-2725; DOI: 10.1021/ jf803633f.

6. N. Ihme, H. Kiesewetter, F. Jung, K. H. Hoffmann, A. Birk, A. Muller and K. I. Grutzner, Leg oedema protection from a buckwheat herb tea in patients with chronic venous insufficiency: a single centre, randomised, double blind, placebo-controlled clinical trial, Eur. J. Clin. Pharmacol. 50 (1996) 443-447; DOI: 10.1007/s002280050138.

7. B. Archimowicz Cyrylowska, B. Adamek, M. Drozdzik, L. Samochowiec and J. Wojcicki, Clinical effect of buckwheat herb, Ruscus extract and troxerutin on retinopathy and lipids in diabetic patients, Phytother. Res. 10 (1998) 659-662; DOI: 10.1002/(SICI)1099-1573(199612)10:8<659::AIDPTR930>3.0.CO;2-U.

8. C. L. Liu, Y. S. Chen, J. H. Yang, B. H. Chiang and C. K. Hsu, Trace element water improves the antioxidant activity of buckwheat (Fagopyrum esculentum Moench) sprouts, J. Agric. Food Chem. 55 (2007) 8934-8940; DOI: 10.1021/jf0716275.

9. S. J. Kim, T. Maeda, M. Z. Sarker, S. Takigawa, C. Matsuura-Endo, H. Yamauchi, Y. Mukasa, K. Saito, N. Hashimoto, T. Noda, T. Saito and T. Suzuki, Identification of anthocyanins in the sprouts of buckwheat, J. Agric. Food Chem. 55 (2007) 6314-6318; DOI: 10.1021/jf0704716.

10. B. Habermann, Protofagopyrin or fagopyrin, what is genuine?, Arch. Pharm. Pharm. Med. Chem. 333 (Suppl. 2) (2000) 13.

11. K. Eguchi, T. Anase and H. Osuga, Development of a high-performance liquid chromatography method to determine the fagopyrin content of tartary buckwheat (Fagopyrum tartaricum Gaertn.) 
and common buckwheat (F. esculentum Moench), Plant Prod. Sci. 12 (2009) 475-480; DOI: $10.1626 /$ pps.12.475.

12. R. Ebermann, G. Alth, M. Kreitner and A. Kubin, Natural products derived from plants as potential drugs for the photodynamic destruction of tumor cells, J. Photochem. Photobiol. B 36 (1996) 95-97; DOI: 10.1016/S1011-1344(96)07353-8.

13. L. Ožbolt, S. Kreft, I. Kreft, M. Germ and V. Stibilj, Distribution of selenium and phenolics in buckwheat plants grown from seeds soaked in Se solution and under different levels of UV-B radiation, Food Chem. 110 (2008) 691-696; DOI: 10.1016/j.foodchem.2008.02.073.

14. C. Theurer, K. I. Gruetzner, S. J. Freeman and U. Koetter, In vitro phototoxicity of hypericin, fagopyrin rich, and fagopyrin free buckwheat herb extracts, Pharm. Pharmacol. Lett. 7 (1997) 113-115.

15. J. Brockmoller, T. Reum, S. Bauer, R. Kerb, W. D. Hubner and I. Roots, Hypericin and pseudohypericin: pharmacokinetics and effects on photosensitivity in humans, Pharmacopsychiatry 30 (Suppl. 2) (1997) 94-101; DOI: 10.1055/s-2007-979527.

16. R. M. Gulick, V. McAuliffe, J. Holden-Wiltse, C. Crumpacker, L. Liebes, D. S. Stein, P. Meehan, S. Hussey, J. Forcht and F. T. Valentine, Phase I studies of hypericin, the active compound in St. John's Wort, as an antiretroviral agent in HIV-infected adults. AIDS Clinical Trials Group Protocols 150 and 258, Ann. Intern. Med. 130 (1999) 510-514; DOI: 10.7326/0003-4819-130-6-1999 03160-00015.

17. H. Chick and P. Ellinger, The photo-sensitizing action of buckwheat (Fagopyrum esculentum), J. Physiol. 100 (1941) 212-230.

18. N. Fabjan, J. Rode, I. J. Kosir, Z. H. Wang, Z. Zhang and I. Kreft, Tartary buckwheat (Fagopyrum tataricum Gaertn.) as a source of dietary rutin and quercitrin, J. Agric. Food Chem. 51 (2003) 64526455; DOI: 10.1021/jf034543e.

19. S.-J. Kim, I. S. M. Zaidul, T. Suzuki, Y. Mukasa, N. Hashimoto, S. Takigawa, T. Noda, C. Matsuura-Endo and $\mathrm{H}$. Yamauchi, Comparison of phenolic compositions between common and tartary buckwheat (Fagopyrum) sprouts, Food Chem. 110 (2008) 814-820; DOI: 10.1016/j.foodchem. 2008.02.050. 